\title{
Structure-Property Relation in Varieties of Silk Fibers
}

\author{
V. ANNADURAI, ${ }^{1}$ G. SUBRAMANYAM, ${ }^{2}$ R. GOPALKRISHNE URS, ${ }^{3}$ R. SOMASHEKAR ${ }^{4}$ \\ ${ }^{1}$ Department of Physics, BIET, Davanagere-577 004, India \\ ${ }^{2}$ Department of Studies in Sericulture, University of Mysore, Manasagangotri, Mysore-570 006, India \\ ${ }^{3}$ Department of Applied Physics, NIE, Mysore-570 008, India \\ ${ }^{4}$ Department of Studies in Physics, University of Mysore, Manasagangotri, Mysore-570 006, India
}

Received 30 November 1999; accepted 29 March 2000

\begin{abstract}
Crystallite shape ellipsoid in different varieties of silk fibers namely (i) Chinese (ii) Indian, and (iii) Japanese, has been computed using wide-angle X-ray data and Hosemann's one-dimensional paracrystalline model. The estimated microcrystalline parameters are correlated with the observed physical property of the silk fibers. (C) 2001 John Wiley \& Sons, Inc. J Appl Polym Sci 79: 1979-1985, 2001
\end{abstract}

Key words: $\quad$ silk fibers; paracrystalline model; wide-angle X-ray data

\section{INTRODUCTION}

Silk fiber is a semicrystalline natural polymer. ${ }^{1-3}$ With the advent of bioengineering, new races and, hence, new varieties of silk fibers are being introduced to the textile industry. This has led to a continued interest in the study of microstructural parameter characterization of these fibers. ${ }^{4-6}$ In this article, we have carried out X-ray profile analysis of two equatorial reflections observed in varieties of silk fibers to compute the crystal imperfection parameters and, hence, the shape of crystallites. The variation of these microstructural parameters is compared with the changes in physical properties like tensile strength and percentage of elongation at break of the silk fibers.

Correspondence to: R. Somashekar.

Contract grant sponsor: UGC.

Journal of Applied Polymer Science, Vol. 79, 1979-1985 (2001)

(C) 2001 John Wiley \& Sons, Inc.

\section{EXPERIMENTAL}

\section{Sample Preparation}

Cocoons are the raw product to reel silk fibers. Hence, cocoons of various races Chinese, Indian, and Japanese were collected from the germplasm stock of the Department of Sericulture and were reeled following the standard procedure. First cocoons were cooked in boiling water for $2 \mathrm{~min}$ to soften the sericin and later transferred to waterbath at $65^{\circ} \mathrm{C}$ for $2 \mathrm{~min}$. Then the cocoons were reeled in warm water with the help of reeling equipment known as Epprouvite. Among the Chinese races, there are two different types, identified as C108 and C145. There are five different types, namely HM (Hosa Mysore), KA (Kalimpong A), $N B_{18}, N B_{7}$, and Nistari among Indian types. In Japanese races, there are three different types, namely Diazo, J106, and J115. It is to be noted that the races HM, Nistari, and Diazo are produced several times in a year and, hence, these fibers are also being referred to as Multivoltine (MV). C108, C145, $\mathrm{KA}, N B_{18}, N B_{7}, \mathrm{~J} 106$, and $\mathrm{J} 115$ are the races pro- 
Table I Characteristic Physical Properties of Varieties of Silk Fibers

\begin{tabular}{|c|c|c|c|c|c|c|}
\hline Sample (Race) & $\begin{array}{l}\text { Cocoon } \\
\text { Shape }\end{array}$ & Origin & Color & Denier & $\begin{array}{l}\text { Tensile } \\
\text { Strength } \\
(\mathrm{MPa})\end{array}$ & $\begin{array}{c}\% \text { of } \\
\text { Elongation } \\
\text { at Break }\end{array}$ \\
\hline C108 & Oval & Chinese & White & $2.2-2.4$ & $65 \pm 1$ & $40.0 \pm 5.7$ \\
\hline C145 & Oval & Chinese & White & $2.2-2.4$ & $118 \pm 9$ & $37.0 \pm 1.9$ \\
\hline HM (Hosa Mysore) & Oval & $\begin{array}{l}\text { Indian } \\
\quad(\text { Karnataka })\end{array}$ & Light green & 2 & $340 \pm 26$ & $33.3 \pm 3.5$ \\
\hline KA (Kalimpong A) & Oval & Indian & White & $2.0-2.2$ & $122 \pm 6$ & $21.2 \pm 1$ \\
\hline$N B_{18}$ & Dumble & Indian & White & 2.1 & $118 \pm 3$ & $24.7 \pm 0.6$ \\
\hline$N B_{7}$ & Oval & Indian & White & 2.1 & $203 \pm 12$ & $24.4 \pm 1.9$ \\
\hline Nistari & Spindle & $\begin{array}{l}\text { Indian } \\
\text { (West bengal) }\end{array}$ & Orange & 1.8 & $135 \pm 12$ & $21.0 \pm 1.6$ \\
\hline Diazo & Spindle & Japanese & Green & $1.9-2.0$ & $141 \pm 4$ & $18.7 \pm 0.5$ \\
\hline J106 & Dumble & Japanese & White & $2.3-2.5$ & $154 \pm 6$ & $21.3 \pm 0.5$ \\
\hline $\mathrm{J} 115$ & Dumble & Japanese & White & $2.3-2.5$ & $171 \pm 8$ & $25.3 \pm 2.5$ \\
\hline
\end{tabular}

duced only twice a year, and hence, are called Bivoltine (BV). These notations are being commonly used by sericulture scientists. The mechanical properties, such as tensile strength and percentage of elongation at break, were measured as per the ASTM D-882 method using a Hounsfield Universal Testing Machine (UTM), and standard deviations are also given. The characteristic physical properties of these silk fibers are given in Table I.

\section{X-ray Recording}

X-ray intensity data for profile analysis from silk fibers was collected on a STOE/STADI-P powder diffractometer with Bragg-Brentano geometry (fine focus setting) with germanium monochromated $\mathrm{CuK}_{\alpha},(\lambda=1.5406 \AA)$ radiation in $2 \theta$ range $5^{\circ}$ to $40^{\circ}$ at intervals of $0.03^{\circ}$ using a curved position sensitive detector (CPSD). The output pattern was corrected for (i) Lorentz polarization factor and (ii) instrumental broadening using the Stokes deconvolution method. ${ }^{7}$ These corrected X-ray profiles were used for further analysis [see Fig. 1(a)-(c)].

\section{X-ray Profile Analysis}

The crystal imperfection parameters like crystal size $(\langle N\rangle)$ and lattice strain $(g)$ were determined by employing the Fourier method of Warren, ${ }^{8,9}$ and Hosemann's one-dimensional paracrystalline model where the simulated intensity profile was matched with the experimental one. ${ }^{10}$ The following equations were employed to simulate the wide-angle X-ray profile.

$$
I(s)=\sum_{n=-\infty}^{\infty} A(n) \cos \left\{2 \pi n d\left(s-s_{o}\right)\right\}
$$

where $A(n)$ is the product of size coefficients $A_{s}(n)$ and lattice distortion (strain coefficient) $A_{d}(n)$. The expression and explanation of the various notations are given in our earlier article. ${ }^{11}$ Using Hosemann's one-dimensional paracrystalline model we have the following relations ${ }^{10}$

$$
I(s)=I_{N-1}(s)+I_{N}(s)
$$

where

$$
\begin{aligned}
I_{N}(s)=2 * \operatorname{Re}\left[\frac{\left(1-I^{N+1}\right)}{(1-I)}+\frac{I v}{d(1-I)^{2}}\right. & \\
\left\{I^{N}(N(1-I)+1)-1\right\} & ]^{-1}
\end{aligned}
$$

where

$$
\begin{aligned}
v & =2 i a^{2} s+d \quad \text { and } \quad I=I_{1}(s) \\
& =\exp \left(-a^{2} s^{2}+i d s\right) ; a^{2}=\omega^{2} / 2
\end{aligned}
$$

Also,

$$
\begin{aligned}
& I_{N}(s)=\frac{2 a_{N}}{D(\pi)^{1 / 2}} \exp (i D s) {\left[1-a_{N} s\left\{2 \mathscr{D}\left(a_{N} s\right)\right.\right.} \\
&\left.\left.+i(\pi)^{1 / 2} \exp \left(-a_{N}^{2} s^{2}\right)\right\}\right]
\end{aligned}
$$



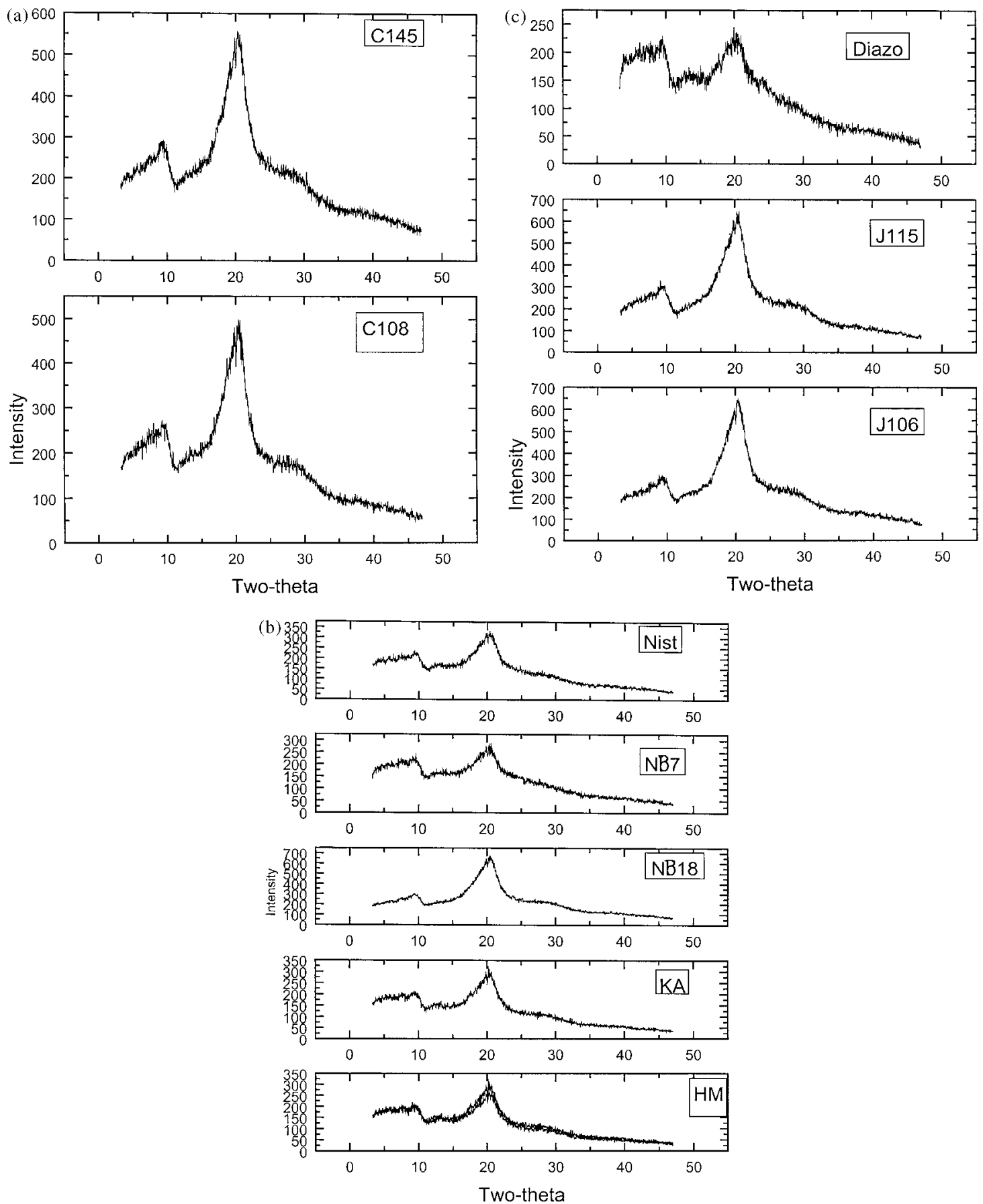

Figure 1 Wide-angle X-ray scattering patterns for (a) Chinese (b) Indian, and (c) Japanese silk fibers.

with $a_{N}^{2}=N \omega^{2} / 2, \omega$ is the standard deviation of the nearest-neighbor probability function, $\mathscr{D}\left(a_{N} s\right)$ is the Dawson's integral or the error function with complex argument, and can be computed. $\langle N\rangle$ is the number of unit cells counted in a direction perpendicular to the $(h k l)$ Bragg plane, $d$ is the spacing of the $(h k l)$ planes, $R e$ refers to the real part of the expression, $s$ is $\sin \theta / \lambda, \lambda$ is the wavelength of X-rays used, $a$ is related to the standard deviation $\omega$ of lattice distribution function, and $D$ is the crystal size $\left(=\langle N\rangle d_{h k l}\right) . I_{N}(s)$ is the modified intensity for the probability peak centered at $D$. 

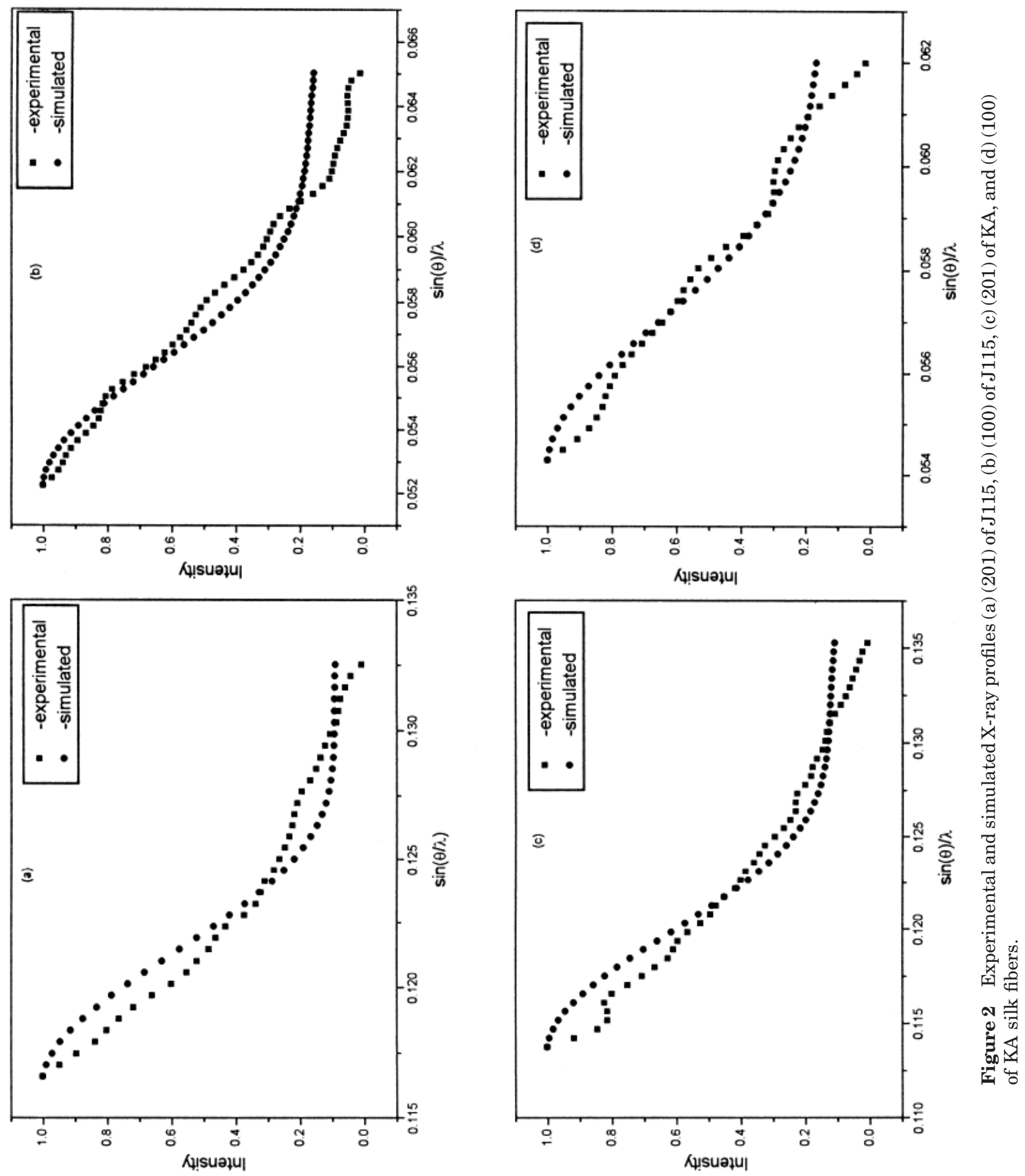
Table II Microstructural Parameters of Different Silk Fibers Obtained with (100) and (201) X-ray Reflection

\begin{tabular}{lccrc}
\hline \multicolumn{1}{c}{ Sample (Race) } & Reflection (hkl) & $\langle N\rangle$ & \multicolumn{1}{c}{$g$ in $\%$} & $D_{s}$ in \\
\hline C108 & & & \\
& $(100)$ & $4.2 \pm 0.1$ & $12.1 \pm 0.2$ & 40.69 \\
C145 & $(201)$ & $5.6 \pm 0.1$ & $7.4 \pm 0.1$ & 24.58 \\
& $(100)$ & $4.8 \pm 0.1$ & $9.1 \pm 0.1$ & 44.25 \\
HM (Hosa Mysore) & $(201)$ & $5.5 \pm 0.1$ & $7.4 \pm 0.1$ & 24.01 \\
& $(100)$ & $4.0 \pm 0.1$ & $9.0 \pm 0.1$ & 39.76 \\
KA (Kalimpong A) & $(201)$ & $6.6 \pm 0.1$ & $7.5 \pm 0.1$ & 28.61 \\
& $(100)$ & $5.9 \pm 0.1$ & $10.6 \pm 0.1$ & 54.87 \\
$N B_{18}$ & $(201)$ & $5.4 \pm 0.1$ & $9.5 \pm 0.1$ & 23.61 \\
& $(100)$ & $5.7 \pm 0.1$ & $11.0 \pm 0.1$ & 53.66 \\
$N B_{7}$ & $(201)$ & $5.9 \pm 0.1$ & $9.2 \pm 0.1$ & 25.68 \\
Nistari & $(100)$ & $8.3 \pm 0.1$ & $11.0 \pm 0.1$ & 75.33 \\
& $(201)$ & $4.7 \pm 0.1$ & $6.3 \pm 0.1$ & 19.98 \\
Diazo & $(100)$ & $5.2 \pm 0.1$ & $9.6 \pm 0.1$ & 47.27 \\
& $(201)$ & $5.4 \pm 0.1$ & $7.8 \pm 0.1$ & 23.06 \\
J106 & $(100)$ & $4.6 \pm 0.1$ & $7.6 \pm 0.1$ & 42.38 \\
& $(201)$ & $4.8 \pm 0.1$ & $6.9 \pm 0.1$ & 20.69 \\
J115 & $(100)$ & $3.2 \pm 0.1$ & $12.3 \pm 0.1$ & 31.09 \\
& $(201)$ & $5.8 \pm 0.1$ & $7.1 \pm 0.1$ & 25.26 \\
& $(100)$ & $4.0 \pm 0.1$ & $7.9 \pm 0.1$ & 38.55 \\
& $(201)$ & $5.9 \pm 0.1$ & $6.5 \pm 0.1$ & 25.24 \\
\hline
\end{tabular}

\section{Computation and Discussion}

The experimental profile between $s_{0}$ and $s_{0}$ $+s_{0} / 2$ is matched with the corresponding simulating order of reflection for various values of $\langle N\rangle$ and $g$ using eqs. (2)-(4) to minimize the difference between calculated and experimental normalized intensity values. Here $s_{0}$ is the value of $s(=\sin$ $\theta / \lambda)$ at the peak of reflection profile. SIMPLEX, a multidimensional algorithm, ${ }^{12}$ is used for minimization. Initial $\langle N\rangle$ crystal size value and width of crystal size distortion $(\alpha)$ were determined using a well-established procedure. $^{9}$ The surface weighted crystal size value $D_{s}$ was also determined using the parameters $\langle N\rangle$ and $\alpha$. The relation is given by

$$
D_{s}=\frac{\int_{0}^{\infty} L P_{s}(L) d L}{\int_{0}^{\infty} P_{s}(L) d L}
$$

where $P_{s}$ is crystal size distribution function, and $L=n d_{h k l}, n$ is the harmonic number. ${ }^{13}$ For the sake of completeness, we have reproduced the simulated and experimental profiles for some of the samples used here [see Fig. 2(a)-(d)].

The microstructural parameters obtained for various silk fibers using two available reflections (100) and (201) are given in Table II. It is evident from the Table II that strains present in these fibers vary between 6.3 to $12.3 \%$.

A graphical plot of the crystallite shape ellipsoid was obtained by taking the crystal size value corresponding to (100) direction along the $\mathrm{X}$-axis and the other parameter corresponding to the (201) direction along the Y-axis [see Fig. 3(a-b)]. From this it is evident that there are significant changes only in the periphery of the crystallite shape ellipsoid.

Figure $4(a-b)$ shows the variation of tensile strength and percentage of elongation at break with crystal size obtained for different silk fibers studied here. In this, we observe that there is a positive slope indicating even a small $1.5 \%$ variation in crystal size value leads to 11.2 and $1.5 \%$ changes in tensile strength and percentage of elongation at break, respectively. Similar changes were earlier reported ${ }^{14}$ for man-made fibers. 


\section{CONCLUSIONS}

From these studies we observe that HM [Hosa Mysore, Indian (MV)] silk fiber has a higher crystal size value and, hence, correspondingly higher tensile strength and percentage of elongation at break compared to other varieties of silk fibers. The significant differences in
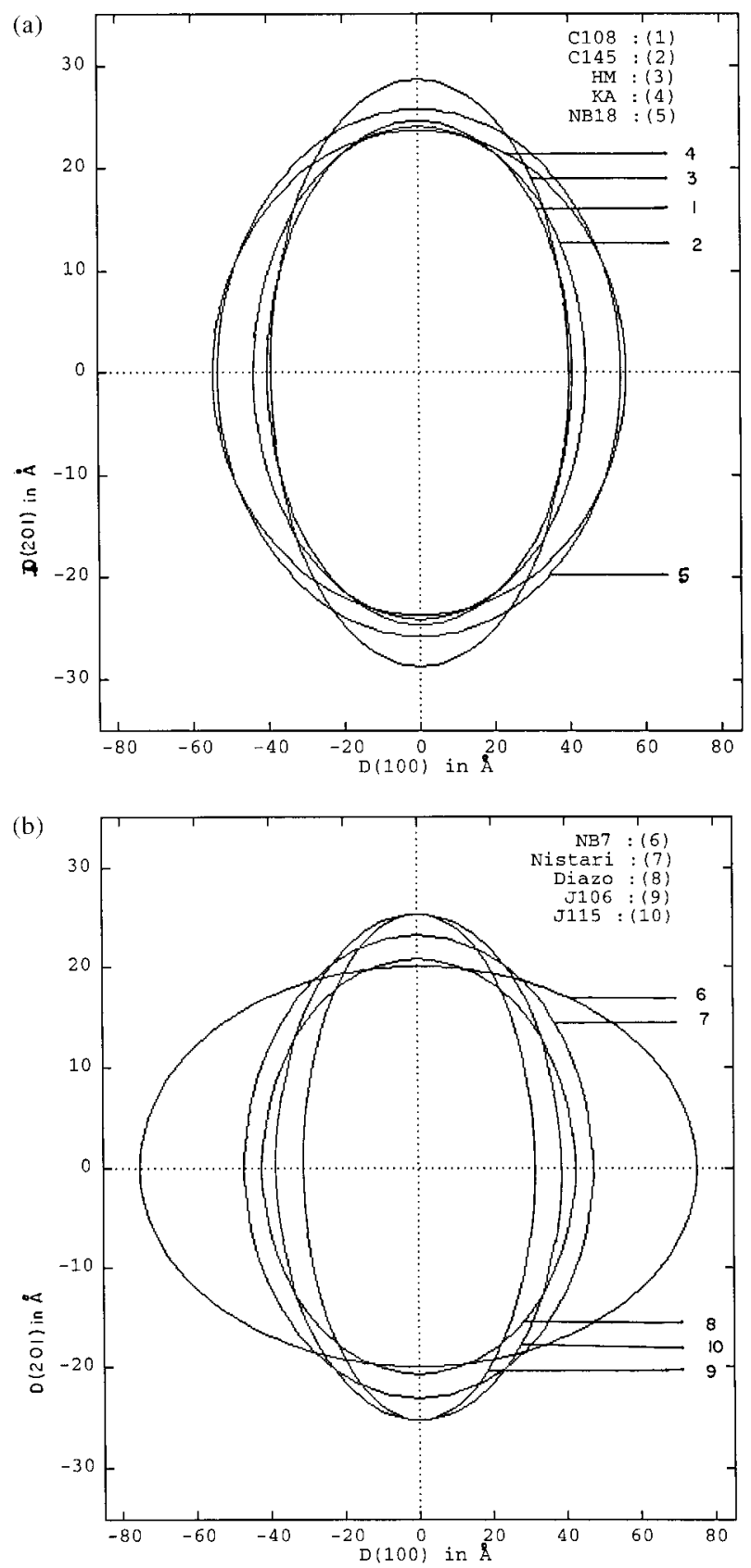

Figure $3(a-b)$ Variation of crystallite shape ellipsoid for different silk fibers.
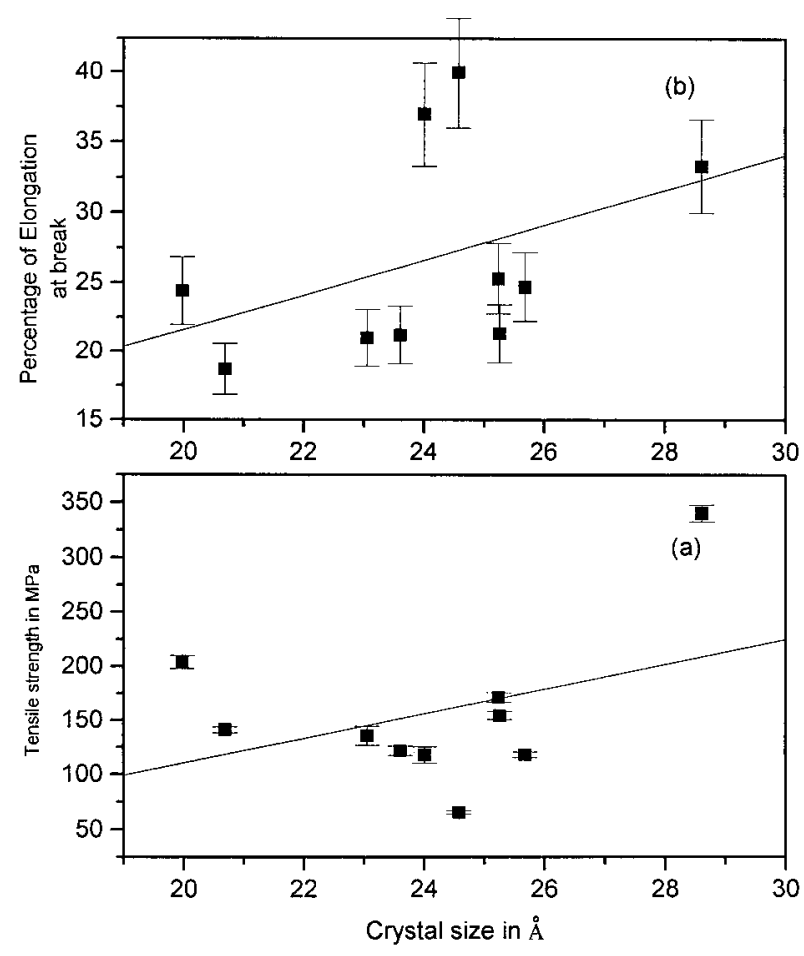

Figure 4 (a-b) Variation of tensile strength and percentage of elongation at break with crystal size observed in silk fibers.

microcrystalline parameters among various types of silk fibers may be attributed to the variation in the fibroin content in the races under study.

The authors thank UGC for financial assistance. One of us (VA) thanks the Principal and the management of Bapuji Institute of Engg and Tech., Davanagere for the encouragement. Dr. R. G. Urs thanks the Principal, NIE, Mysore for the facilities.

\section{REFERENCES}

1. Marsh, R. E.; Corey, R. B.; Pauling, L. Biochem Biophys Acta 1955, 16, 1.

2. Demura, M.; Minami, M.; Asakura, T.; Cross, T. A. J Am Chem Soc 1998, 120, 1300.

3. Kenji, O.; Katsuhiro, T.; Yashushi, N.; Yoshiko, H.; Kiyoshi, H.; Norio, N. J Seric Sci 1992, 57, 23.

4. Somashekar, R.; Gopalkrishne Urs, R.; Madhava, M. S. J Appl Polym Sci 1992, 44, 2161.

5. Somashekar, R.; Gopalkrishne Urs, R. Polymer 1995, 36, 2007. 
6. Somashekarappa, H.; Nadiger, G. S.; Somashekar, T. H.; Prabhu, J.; Somashekar, R. Polymer 1997, $39,209$.

7. Stokes, A. R. Proc Phys Soc Lond 1948, 61, 382.

8. Somashekar, R.; Hall, I. H.; Carr, P. D. J Appl Crystallogr 1989, 32, 363.

9. Hall, I. H.; Somashekar, R. J Appl Crystallogr 1991, 24, 1051.

10. Somashekar, R.; Somashekarappa, H. J Appl Crystallogr 1997, 30, 147.
11. Somshekarappa, H.; Somashekar, R.; Vasudev, S.; Ali, S. Z. Bull Mater Sci 1999, 22, 101.

12. Press, W.; Flannery, B. P.; Teuklosky, S.; Vettering, W. T. Numerical Recipes; Cambridge University Press: New York, 1988, p. 284.

13. Somashekar, R.; Somashekarappa, H.; Subramaniam, G.; Prahllad, U. D. Eur Polym J 1997, 33, 963.

14. Lee, K. G.; Barton, R. Jr.; Schultz, J. M. J Polym Sci (B) 1995, 33, 1. 